\section{Isolare i pazienti HBsAg? Una pratica di medicina difensiva}

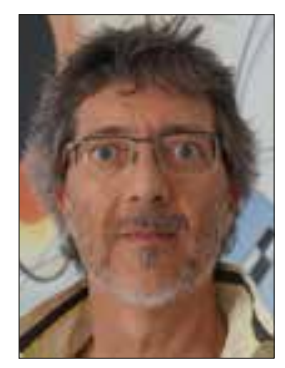

Marco Lombardi

\section{Chiar.mo Dr. Gennaro Santorelli,}

Sono conscio del fatto che il mio articolo sulla poca utilità della contumaciazione in dialisi per la prevenzione della diffusione delle infezioni da virus ematogeni (1) possa essere letto come una provocazione, perché di fatto lo è, anche se chi ha scritto nel 2009 l'articolo sul GIN (2) crede davvero a quanto afferma e in tal senso rimando anche alla lettera apparsa sullo stesso numero del giornale (3).

Perché penso che per ridurre o azzerare la diffusione del virus dell'epatite $B$ in dialisi non sia necessario l'isolamento dei pazienti HBsAg positivi? L'ho scritto in detto articolo ma provo a convincerla con poche semplici argomentazioni.

A fronte di quanto storicamente raccomandato dai CDC di Atlanta (isolamento dei pazienti HBsAg+ tramite l'utilizzo di sale contumaciali, macchinari dedicati e personale dedicato), la letteratura fornisce pochi dati a favore dell'utilità di questa politica di isolamento e purtroppo pochi sono anche i dati a favore dell'ipotesi contraria; insomma, come scrive lei stesso nella sua lettera (4), "questo argomento resta estremamente dibattuto e di grande attualità perché non si hanno certezze in merito e $i$ dati epidemiologici a favore di una o dell'altra evidenza non sono tanti". Questo a mio avviso è dettato viceversa da un dibattito insufficiente e dalla difficoltà di attuare (e quindi reperire nella letteratura) studi controllati per confrontare in modo serio le due ipotesi. Come se non bastasse la povertà di studi controllati produce nel nostro Paese un'altra difficoltà di non poco conto: non esiste una regolamentazione NAZIONALE e chi è interessato può reperire solo regolamentazioni REGIONALI, spesso e volentieri le più recenti scopiazzate dalle meno recenti, sconclusionate e/o comunque poco affidabili... ove si raccomanda per esempio di contumaciare/separare/dedicare non solo i pazienti $\mathrm{HBsAg}+$ ma anche gli HCV+ e gli HIV+. Da questa che considero un'inappropriatezza delle regolamentazioni regionali si capisce chiaramente che al legislatore poco è importato (o non le conosceva?) delle caratteristiche peculiarmente "cattive" del virus dell'epatite B rispetto agli altri due principali patogeni ematogeni, razionale che lei nella sua lettera, così come i CDC nei loro report, porta a supporto dell'ipotesi favorevole a contumaciare: se il legislatore avesse considerato importante questa differenza non avrebbe certo obbligato a contumaciare/separare/ dedicare anche i pazienti HCV/HIV+! Forse è valsa la regola del Melius abundare quam deficere (o, come diciamo noi in Toscana, meglio aver paura che buscarne)? Può darsi, in tutto si può trovare un razionale; ma quanto si confà questo dettato alla problematica di sostenibilità economica dei nostri tempi correnti?

Restando alle caratteristiche malefiche dell'HBV (I'ubiquità e la resistenza e la carica virale elevate), anche se rivestono grande importanza, non possiamo non considerare che sappiamo che queste possono essere controllate da una corretta e soprattutto continua applicazione delle pratiche descritte nelle Precauzioni Universali e Speciali (PU/S). Per esempio trovo inaccettabile che, sapendo che la modalità di trasmissione del virus dell'epatite $B$ in dialisi è tendenzialmente di tipo parenterale-inapparente, il passaggio, come lei scrive, possa essere veicolato dai guanti di un infermiere: per questo la contumacia non serve a niente, nel senso che è moralmente e concettualmente errato ricorrere a una misura effettivamente eccessiva e inadeguata, per una malpractice che potrebbe/dovrebbe essere sanata con l'educazione del personale e con l'adozione di misure assai più semplici da realizzare (alias $\mathrm{PU} / \mathrm{S}$ ) rispetto alla contumacia: basterebbe imparare a cambiarsi sempre i guanti! La stessa identica cosa vale per l'eventuale presenza del (o dei) virus su forbici, clamp e comandi dei monitor di dialisi, cosa possibile solo se le PU/S (lavaggio, disinfezione, ecc.) non vengono messe in atto correttamente e pedissequamente!

Per quanto attiene viceversa al rischio di trasmissione del (o dei) virus per il contagio attraverso la macchina mediante i trasduttori di pressione, non mi risulta che vi siano lavori attendibili che lo dimostrino chiaramente (purtroppo o per fortuna?): le ditte ci propongono una sorta di medicina difensiva più a favore loro che dei pazienti.

Concluderei ponendo il seguente quesito a lei ma anche ai lettori di questa mia risposta: consci che l'errore è insito nello svolgimento delle pratiche in stanza di dialisi e che non è completamente eliminabile, per prevenire nuove infezioni è preferibile che l'investimento necessario sia speso per la creazione di (pochi) centri contumaciali (in ospedali ad hoc per porzioni di territorio più o meno ampie) o viceversa che sia speso per ampliare l'educazione e la conoscenza delle $\mathrm{PU} / \mathrm{S}$ affinché tutto il personale le applichi tutte e in tutte le manovre assistenziali... e, perché no, affinché le si diffondano anche a tutti i pazienti (regola delle 3-4 T)? (1).

Certamente sappiamo tutti che è molto più semplice e di facciata avere un centro contumaciale ove scaricare i pazienti positivi che avere un personale reso tutto costantemente abile alla messa in opera delle PU/S.

Per questo auspico e mi piacerebbe ottenere il parere della branca infermieristica, che mi sento di chiamare in causa perché costantemente in prima linea nei nostri centri dialisi.

\section{Disclosures}

Financial support: No financial support was received for this submission. Conflict of interest: The author has no conflict of interest. 


\section{Marco Lombardi}

S.C. Nefrologia e Dialisi, Ospedale S.M. Annunziata e S.S. Dialisi del Mugello, Azienda Sanitaria di Firenze, Firenze

Indirizzo per la corrispondenza:

Dr. Marco Lombardi

S.S. Dialisi del Mugello

Via della Resistenza

50032 Borgo S. Lorenzo (FI)

lombardim@tin.it

\section{Bibliografia}

1. Lombardi M. II punto su epatite B e C in dialisi: riflessioni sulla contumacia dei pazienti. Giornale di Tecniche Nefrologiche e Dialitiche 2014;26(4):321-5.

2. Lombardi M. Cherubini C. La mancata osservanza di tutte le precauzioni speciali è un reale rischio clinico in dialisi. G Ital Nefrol 2009;25(5):625-31.

3. Cherubini C. Il senso della contumacia (lettera). Giornale di Tecniche Nefrologiche e Dialitiche 2014;26(4):398.

4. Santarelli G. L'isolamento in emodialisi: una questione aperta. Giornale di Tecniche Nefrologiche e Dialitiche 2015;27(1): 63-64.

Published online: February 26, 2015 